\title{
Tree Embeddings and Tree-Star Ramsey Numbers
}

\author{
Zilong Yan * Yuejian Peng ${ }^{\dagger}$
}

January 3, 2022

\begin{abstract}
We say that a graph $F$ can be embedded into a graph $G$ if $G$ contains an isomorphic copy of $F$ as a subgraph. Guo and Volkmann [6] conjectured that if $G$ is a connected graph with at least $n$ vertices and minimum degree at least $n-3$, then any tree with $n$ vertices and maximum degree at most $n-4$ can be embedded into $G$. In this paper, we give a result slightly stronger than this conjecture and obtain a sufficient and necessary condition that a tree with $n$ vertices and maximum degree at most $n-3$ can be embedded into a connected graph $\mathrm{G}$ with at least $n$ vertices and minimum degree at least $n-3$. Our result implies that the conjecture of Guo and Volkmann is true with one exception. We also give an application to the Ramsey number of a tree versus a star.
\end{abstract}

Keywords: Tree, Embedding, Ramsey number, Tree-Star Ramsey number.

\section{Introduction}

All graphs considered throughout the paper are simple graphs, i.e. without loops and multiple edges. Let $V(G)$ denote the vertex set of $G$ and let $E(G)$ denote the edge set of $G$. For $v \in V(G)$, let $N(v)=\{u \in$ $V(G) \mid u v \in E(G)\}, N[v]=N(v) \cup\{v\}$, and $d(v)=|N(v)|$. For $S \subseteq V(G)$, denote $N(S)=\cup_{v \in S} N(v)$ and $N[S]=N(S) \cup S$. Let $\delta(G)=\min \{d(v) \mid v \in V(G)\}$ and $\Delta(G)=\max \{d(v) \mid v \in V(G)\}$. Let $K_{n}$ denote the complete graph on $\mathrm{n}$ vertices and $K_{1, m}$ denote the star with $m+1$ vertices. For graphs $G$ and $H$, the Ramsey Number $R(G, H)$ is the smallest integer $N$ such that any red-blue-coloring of $E\left(K_{N}\right)$ yields a red $G$ or a blue $H$. For graphs $F$ and $G$, we say that an injection $\phi: V(F) \rightarrow V(G)$ is an embedding of $F$ into $G$ if for any edge $x y$ in $F, \phi(x) \phi(y)$ is an edge in $G$. We say that $F$ can be embedded into $G$ if there is an embedding of $F$ into $G$.

Degree conditions for tree embedding have been studied actively. A well-known conjecture of ErdősSós states that any tree with $n$ vertices can be embedded into a simple graph with average degree exceeding $n-2$. In [7, Havet, Reed, Stein and Wood studied the conditions on the maximum degree and the minimum degree of a graph to embed any tree with $n$ vertices, they proposed an interesting conjecture that any tree with $n$ vertices can be embedded into a simple graph with maximum degree more than $n+1$ and minimum degree at least $\left\lfloor\frac{2(n+1)}{3}\right\rfloor$. The Loebl-Komlós-Sós conjecture [5] states that any tree with $n$ vertices can be embedded into a simple graph with median degree at least $n+1$. We

\footnotetext{
*School of Mathematics, Hunan University, Changsha 410082, P.R. China. Email: zilongyan@hnu.edu.cn.

${ }^{\dagger}$ Corresponding author. School of Mathematics, Hunan University, Changsha, 410082, P.R. China. Email: ypeng1@hnu.edu.cn. Supported in part by National Natural Science Foundation of China (No. 11931002).
} 
study minimum degree conditions to embed trees, and it has natural applications on tree-star Ramsey numbers.

In $\left[\right.$, Parson determined the path-star Ramsey numbers. A key ingredient in the proof is that $P_{n}$, a path with $n$ vertices, can be embedded into a graph with minimum degree $n-1$. This is generalized to the following well-known result (see [3]).

Lemma 1.1 If $G$ is a graph with minimum degree $n-1$, then any tree with $n$ vertices can be embedded into $G$.

This lemma implies the Ramsey result of Burr [2]: $R\left(T_{n}, K_{1, m}\right) \leq m+n-1$, where $T_{n}$ is a tree with $n$ vertices and $K_{1, m}$ is the star with $m+1$ vertices. Applying Lemma 1.1. Cockayne 3 improved the upper bound of $R\left(T_{n}, K_{1, m}\right)$ to $m+n-2$ for a special class of trees with $n$ vertices and some values of $m$ and $n$. Further, Guo and Volkmann [6] generalized the result of Cockayne to any tree with $n$ vertices. A key making the generalization possible is that Guo and Volkmann showed that any tree with $n$ vertices other than $K_{1, n-1}$ can be embedded into a connected graph with at least $n$ vertices and minimum degree $n-2$. They remarked that there are connected graphs $G$ with at least $n$ vertices and minimum degree $n-3$, and trees with $n$ vertices and maximum degree $n-3$ which cannot be embedded into $G$. And they proposed the following conjecture.

Conjecture 1 ([6]) If $G$ is a connected graph with at least $n$ vertices and minimum degree at least $n-3$, then any tree with $n$ vertices and maximum degree at most $n-4$ can be embedded into $G$.

We show a result slightly stronger than this conjecture and obtain a sufficient and necessary condition that trees with $n$ vertices and maximum degree at most $n-3$ can be embedded into a graph $G$ with minimum degree at least $n-3$.

Let $p$ and $q$ be positive integers. Let $T(p, q)$ be a tree with a longest path $v_{1} v_{2} v_{3} v_{4} v_{5}$ satisfying that $d\left(v_{3}\right)=2$, and $v_{2}$ and $v_{4}$ have $p$ and $q$ leaves respectively (see Figure 1 ). The following is the main result

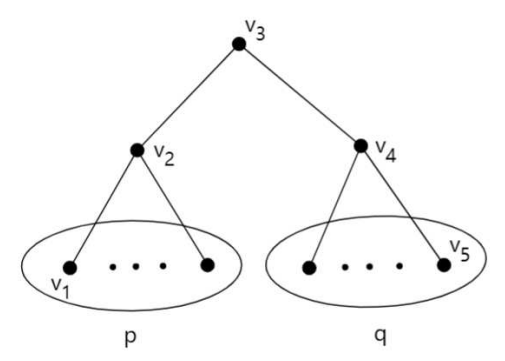

Figure 1: $T(p, q)$

in this paper.

Theorem 1.2 Let $G$ be a connected graph with at least $n$ vertices and minimum degree at least $n-3$. Let $T_{n}$ be a tree with $n$ vertices and $\Delta\left(T_{n}\right) \leq n-3$. Then $T_{n}$ can be embedded into $G$ if and only if the following situations do not happen.

(i) $G=K_{n-3, n-3}$ and $T_{n}=T(p, n-3-p)$, where $p \geq 1$. 
(ii) $G$ is a balanced complete $(k+1)$-partite graph with a vertices in each part and $k a=n-3$, and $T_{n}=T(1, n-4)$, where $k \geq 2$ is a positive integer.

This result implies that Conjecture 1 holds except in situation (i) in Theorem 1.2. A non-negative integer $N$ is called a linear combination of two positive integers $p$ and $q$ if there exist non-negative integers $k$ and $l$ such that $N=k p+l q$. Applying Theorem 1.2, we are going to prove the following tree-star Ramsey numbers in Section 4

Theorem 1.3 Let $T_{n}$ be a tree with $n$ vertices, $\Delta\left(T_{n}\right) \leq n-3$, and $T_{n} \neq T(p, n-3-p)$. If $m+n-3$ is not a linear combination of $n-1$ and $n-2$, then $R\left(T_{n}, K_{1, m}\right) \leq m+n-3$.

Theorem 1.4 Let $T_{n}$ be a tree with $n$ vertices and $\Delta\left(T_{n}\right) \leq n-3$. Let $m=k(n-1)+3$ and $0 \leq k \leq n-5$. (i) If $T_{n} \neq T(p, n-3-p)$, then $R\left(T_{n}, K_{1, m}\right)=m+n-3$.

(ii) If $m+n-3$ is not a linear combination of $n-1, n-2, n-3+a_{1}, \ldots, n-3+a_{d}$, where $a_{1}, a_{2}, \ldots, a_{d}$ are positive divisors of $n-3$ no less than 3 , then $R\left(T(1, n-4), K_{1, m}\right)=m+n-3$. Otherwise $R\left(T(1, n-4), K_{1, m}\right)=m+n-2$.

(iii) Let $p \geq 2$. If $m+n-3$ is not a linear combination of $2 n-6, n-1$ and $n-2$, then $R(T(p, n-3-$ $\left.p), K_{1, m}\right)=m+n-3$. Otherwise $R\left(T(p, n-3-p), K_{1, m}\right)=m+n-2$.

The conjecture of Erdös-Sós states that any tree with $n$ vertices can be embedded into a graph with average degree exceeding $n-2$. It is easy to show that a connected graph with average degree greater than $n-2$ contains a connected subgraph with minimum degree greater than $\frac{1}{2}(n-2)$. So if one can characterize what kind of trees with $n$ vertices can be embedded into a connected graph $G$ with at least $n$ vertices and $\delta(G) \geq \frac{1}{2}(n-1)$, then these trees will satisfy the conjecture. In general, it is interesting to study what kind of $T_{n}$ can be embedded into a connected graph $G$ with at least $n$ vertices and $\delta(G) \geq t$.

In Section 2, we give some crucial lemmas in the proof of Theorem 1.2 , The proof of Theorem 1.2 will be given in Section 3 . In Section 4, we prove Theorems 1.3 to 1.4

\section{Preparations}

Definition 2.1 A labelling $\left\{v_{1}, v_{2}, \ldots, v_{n}\right\}$ of vertices of a tree with $n$ vertices is called a conventional labelling if for each $j \in[2, n],\left|N\left(v_{j}\right) \cap\left\{v_{1}, \ldots, v_{j-1}\right\}\right|=1$. To simplify the notation, we always denote the unique vertex in $N\left(v_{j}\right) \cap\left\{v_{1}, \ldots, v_{j-1}\right\}$ by $v_{j^{\prime}}$ for a conventional labelling.

Note that a tree $T$ always has a conventional labelling. We may take any vertex in $V(T)$ as $v_{1}$ and order the other vertices as $v_{2}, \ldots, v_{n}$ in increasing order of the distances to $v_{1}$.

For given graphs $F$ and $G$, we say that a subset $U \subseteq V(F)$ has been embedded into $G$ (or $\phi$ is an embedding of $U$ to $G$ ) if an embedding $\phi$ of $F[U]$ into $G$ has been established. If $U \subseteq V(F)$ has been embedded to $G$ via an embedding $\phi$, we say that $\phi$ can be extended to a vertex $v \in V(F) \backslash U$ if there exists $v^{\prime} \in V(G)$ such that the extension function $\hat{\phi}: U \cup\{v\} \rightarrow V(G)$ defined by $\hat{\phi}(u)=\phi(u)$ for $u \in U$ and $\hat{\phi}(v)=v^{\prime}$ is an embedding of $F[U \cup\{v\}]$ into $G$.

Lemma 2.2 Let $G$ be a connected graph with at least $n$ vertices. Let $\left\{v_{1}, v_{2}, \ldots, v_{n}\right\}$ be a conventional labelling of $T_{n}$. Let $j \in\{2, \ldots, n\}$. Let $\phi$ be an embedding of $\left\{v_{1}, \ldots, v_{j-1}\right\}$ into $G$.

(i) If

$$
\left|N\left(\phi\left(v_{j^{\prime}}\right)\right) \backslash\left\{\phi\left(v_{1}\right), \ldots, \phi\left(v_{j-1}\right)\right\}\right| \geq 1
$$


then $\phi$ can be extended to $v_{j}$.

(ii) If $\delta(G) \geq n-1$, then $\phi$ can be extended to $v_{j}$.

(iii) Let $\delta(G) \geq n-2$. If $2 \leq j \leq n-1$, then $\phi$ can be extended to $v_{j}$. If $j=n$ and there exists $k \in\{1,2, \ldots, n-1\} \backslash\left\{n^{\prime}\right\}$ such that $\phi\left(v_{k}\right) \notin N\left(\phi\left(v_{n^{\prime}}\right)\right)$, then $\phi$ can be extended to $v_{j}$.

(iv) Let $\delta(G) \geq n-3$. If $2 \leq j \leq n-2$, then $\phi$ can be extended to $v_{j}$. If $j=n-1$ and $\mid\left\{\phi\left(v_{1}\right), \ldots, \phi\left(v_{n-2}\right)\right\} \backslash$ $N\left(\phi\left(v_{(n-1)^{\prime}}\right)\right) \mid \geq 2$, then $\phi$ can be extended to $v_{j}$. If $j=n$ and $\left|\left\{\phi\left(v_{1}\right), \ldots, \phi\left(v_{n-1}\right)\right\} \backslash N\left(\phi\left(v_{n^{\prime}}\right)\right)\right| \geq 3$, then $\phi$ can be extended to $v_{j}$.

Proof of Lemma 2.2. The condition in (i) guarantees that there is a free vertex in $N\left(\phi\left(v_{j^{\prime}}\right)\right)$ to embed $v_{j}$, therefore $v_{j}$ can be embedded to a vertex in $N\left(\phi\left(v_{j^{\prime}}\right)\right)$, and this proves (i). (ii), (iii) and (iv) are direct implications of (i).

Lemma 2.3 (4] Let $G$ be a connected graph with minimum degree $\delta(G)$. Then $G$ contains a path of length $\min \{n, 2 \delta(G)+1\}$.

Lemma 2.4 Let $G$ be a connected graph with minimum degree $\delta$. Let $S_{0} \subseteq V(G)$ and let $S_{1}$ be a proper subset of $S_{0}$ such that $N\left(S_{1}\right) \subseteq S_{0}$. If there exists $\alpha \in S_{0}$ such that $N(\alpha) \not \subset S_{0}$, then there exists a path of length at least $\left|S_{1}\right|+\delta-\left|S_{0}\right|+1$ starting from a neighbor $\alpha$ outside $S_{0}$.

Proof of Lemma 2.4. Let $\alpha_{1} \in N(\alpha) \backslash S_{0}$. Let $\alpha_{1} \alpha_{2} \ldots \alpha_{g}$ be a maximum path outside $S_{0}$. Since $N\left(S_{1}\right) \subseteq S_{0}, N\left(\alpha_{g}\right) \subseteq S_{0} \cup\left\{\alpha_{1}, \alpha_{2}, \ldots, \alpha_{g-1}\right\} \backslash S_{1}$. Since $\left|N\left(a_{g}\right)\right| \geq \delta,\left|S_{0}\right|+g-1-\left|S_{1}\right| \geq \delta$. So $g \geq\left|S_{1}\right|+\delta-\left|S_{0}\right|+1$.

Lemma 2.5 Let $G$ be a non-complete and connected graph, then there exist $\alpha, \beta, \gamma \in V(G)$ such that $\alpha \beta, \alpha \gamma \in E(G)$ and $\beta \gamma \notin E(G)$.

Proof of Lemma 2.5. Take a shortest path between two non-adjacent vertices, then three consecutive vertices in this path satisfy the condition.

\section{$3 \quad$ Embedding trees}

We give the proof of Theorem 1.2 below.

Proof of Theorem 1.2. Let $T_{n}$ be a tree with $n$ vertices. Let $P$ be a longest path of $T_{n}$. Let $|P|$ be the number of vertices of path $P$ and $i=|P|-2$. Since $|P|=3$ implies that $T_{n}=K_{1, n-1}$, it contradicts $\Delta\left(T_{n}\right) \leq n-3$. So $|P| \geq 4$. For $|P| \geq 5$, we label the two end vertices as $v_{n-1}$ and $v_{n}$, all other vertices in $P$ as $v_{1}, v_{2}, \ldots, v_{i}$ such that $P=v_{n-1} v_{1} v_{2} \ldots v_{i} v_{n}$, i.e. $v_{n-1}$ is connected to $v_{1}, v_{1}$ is connected to $v_{2}, \cdots, v_{i-1}$ is connected to $v_{i}$, and $v_{i}$ is connected to $v_{n}$. We order the vertices outside $P$ as $\left\{v_{i+1}, \ldots, v_{n-2}\right\}$ in non-decreasing order of the distances to $P$. For example, $v_{i+1}$ is connected to a vertex in $P$. Note that $\left\{v_{1}, v_{2}, \ldots, v_{n-1}, v_{n}\right\}$ is a conventional labelling. We will show that there is no embedding $\phi: V\left(T_{n}\right) \rightarrow V(G)$ if and only if situation (i) or (ii) in Theorem 1.2 happens.

By Lemma 1.1. we can easily embed $\left\{v_{1}, \ldots, v_{n-2}\right\}$ into $G$. So what we need to work on is to embed $v_{1}$ and $v_{i}$ properly so that there is a free vertex for us to embed $v_{n-1}$ in the neighborhood of the vertex $v_{1}$ embedded to, and there is a free vertex for us to embed $v_{n}$ in the neighborhood of the vertex $v_{i}$ embedded to. 
By Lemma 2.5, there exist $u_{1}, u_{2}$ and $u_{3}$ in $G$ satisfying that $u_{2} \in N\left(u_{1}\right)$ and $u_{3} \in N\left(u_{2}\right) \backslash N\left(u_{1}\right)$. Let $\phi\left(v_{j}\right)=u_{j}$ for $j \in[3]$. By Lemma 2.2 (iv), $\phi$ can be extended to $\left\{v_{4}, \ldots, v_{i-1}\right\}$. Denote $\phi\left(v_{j}\right)=u_{j}$ for $j \in[i-1]$. Denote $K_{i}=\left\{\phi\left(v_{1}\right), \ldots, \phi\left(v_{i-1}\right)\right\}$.

Case 1. $|P| \geq 6$.

Case 1.1. There exists a vertex, denoted by $u_{i}$, in $N\left(u_{i-1}\right) \backslash K_{i}$ such that $\left|K_{i} \backslash N\left(u_{i}\right)\right| \geq 2$.

In this case, let $\phi\left(v_{i}\right)=u_{i}$. Since $\delta(G) \geq n-3, u_{1}, u_{3} \notin N\left(u_{1}\right)$, by Lemma 2.2 (iv), $\phi$ can be extended to $\left\{v_{1}, \ldots, v_{n-1}\right\}$. Further $\left|K_{i} \backslash N\left(u_{i}\right)\right| \geq 2$ implies that $\left|\left\{\phi\left(v_{1}\right), \ldots, \phi\left(v_{n-1}\right)\right\} \backslash N\left(u_{i}\right)\right| \geq 3$, by Lemma 2.2 (iv), there exists an embedding from $T_{n}$ into $G$.

Case 1.2. There exists a vertex, denoted by $u_{i}$, in $N\left(u_{i-1}\right) \backslash K_{i}$ such that $\left|K_{i} \backslash N\left(u_{i}\right)\right|=1$, and $\left|K_{i} \backslash N(u)\right| \leq 1$ for any $u \in N\left(u_{i-1}\right) \backslash K_{i}$.

Note that $\left|K_{i} \backslash N\left(u_{i}\right)\right|=1$ implies that there exists a vertex $u_{x}$ in $K_{i}$ such that $u_{x} u_{i} \notin E(G)$.

If $d\left(u_{i}\right) \geq n-2$, let $\phi\left(v_{i}\right)=u_{i}$. Since $u_{3} \notin N\left(u_{1}\right)$, by Lemma 2.2 (iv), $\phi$ can be extended to $\left\{v_{i+1}, \ldots, v_{n-1}\right\}$. Since $u_{x}, u_{i} \notin N\left(u_{i}\right),\left|N\left(u_{i}\right) \backslash\left\{\phi\left(v_{1}\right), \ldots, \phi\left(v_{n-1}\right)\right\}\right| \geq 1$ and there is at least one free vertex in $N\left(u_{i}\right)$ to embed $v_{n}$. So it is sufficient to consider $d\left(u_{i}\right)=n-3$. Let $K=K_{i} \cup N\left[u_{i}\right]$, then $|K|=n-1$.

Claim 3.1 $N\left(u_{1}\right) \subseteq K$ if $T_{n}$ cannot be embedded into $G$.

Proof of Claim 3.1. Suppose that there exists a vertex denoted by $u_{n-1}$ in $N\left(u_{1}\right) \backslash K$. Since $u_{3} \notin N\left(u_{1}\right)$, by Lemma 2.2 (iv), $\phi$ can be extended to $\left\{v_{1}, \ldots, v_{n-1}\right\}$. Furthermore, we can guarantee that some vertex $v_{a} \in T_{n} \backslash\left\{v_{n}\right\}$ is embedded to $u_{n-1}$, where $a \in[i+1, n-1]$ (This is always possible since we can always embed $v_{n-1}$ to $u_{n-1}$ if $u_{n-1}$ is not previously used). Now $u_{x}, u_{i}, u_{n-1} \notin N\left(u_{i}\right)$, then there exists a free vertex in $N\left(u_{i}\right) \backslash\left\{\phi\left(v_{1}\right), \ldots, \phi\left(v_{n-2}\right), \phi\left(v_{n-1}\right)\right\}$ to embed $v_{n}$ and $T_{n}$ can be embedded into $G$.

Claim 3.2 $N\left(u_{2}\right) \subseteq K$ if $T_{n}$ cannot be embedded into $G$.

Proof of Claim 3.2. If there exists a vertex $u_{1}^{\prime} \in N\left(u_{2}\right) \backslash K$, then reassign $\phi\left(v_{1}\right)=u_{1}^{\prime}$ and let $\phi\left(v_{i}\right)=u_{i}$. By the choice of $u_{1}^{\prime}$, we know that $u_{i} \notin N\left(\phi\left(v_{1}\right)\right)$. By Lemma22.2(iv), $\phi$ can be extended to $\left\{v_{1}, \ldots, v_{n-1}\right\}$. Recall that there exists a vertex $u_{x} \in K_{i}$ such that $u_{x} u_{i} \notin E(G)$. We claim that $u_{x} \neq u_{1}$. Since $d\left(u_{1}\right) \geq n-3, u_{1}, u_{3} \notin N\left(u_{1}\right), N\left(u_{1}\right) \subseteq K$ and $|K|=n-1, u_{1} u_{i} \in E(G)$. Since $u_{x}, u_{1}^{\prime} \notin N\left(u_{i}\right)$, there is at least one free vertex in $N\left(u_{i}\right) \backslash\left\{\phi\left(v_{1}\right), \ldots, \phi\left(v_{n-1}\right)\right\}$ to embed $v_{n}$. A contradiction.

Claim 3.3 $N\left(u_{t}\right) \subseteq K$ for $1 \leq t \leq i-1$ if $T_{n}$ cannot be embedded into $G$.

Proof of Claim 3.3. Use induction on t. For $t=1$ or 2 , it is guaranteed by Claim 3.2. Let $3 \leq t \leq i-1$. We assume that $N\left(u_{s}\right) \subseteq K$ for $1 \leq s \leq t-1$. If $N\left(u_{t}\right) \not \subset K$, then there exists a vertex $u_{t-1}^{\prime} \in N\left(u_{t}\right) \backslash K$. By Lemma 2.4 there exists a path $u_{t-1}^{\prime} u_{t-2}^{\prime} \ldots u_{1}^{\prime}$ outside K. Reassign $\phi\left(v_{\alpha}\right)=u_{\alpha}^{\prime}$ for $1 \leq \alpha \leq t-1$ and let $\phi\left(v_{i}\right)=u_{i}$. Since $u_{\alpha}^{\prime} \notin N\left(u_{i}\right)$ for $1 \leq \alpha \leq t-1$ and $t \geq 3$, by Lemma 2.2 (iv), $\phi$ can be extended to $\left\{v_{i+1}, \ldots, v_{n}\right\}$.

Claim 3.4 $N(K) \subseteq K$ if $T_{n}$ cannot be embedded into $G$.

Proof of Claim 3.4. If there exists a vertex $u_{i-1}^{\prime} \in N\left(u_{i}\right) \backslash K_{i}$ such that there exists a vertex $u_{i-2}^{\prime} \in$ $N\left(u_{i-1}^{\prime}\right) \backslash K$, by Lemma 2.4 , there exists a path $u_{i-2}^{\prime} u_{i-3}^{\prime} \ldots u_{1}^{\prime}$ outside K. Reassign $\phi\left(v_{\alpha}\right)=u_{\alpha}^{\prime}$ for 
$1 \leq \alpha \leq i-1$ and let $\phi\left(v_{i}\right)=u_{i}$. Since $i \geq 4$ guarantees that $u_{1}^{\prime}, u_{2}^{\prime} \notin N\left(u_{i}\right)$, by Lemma 2.2 (iv), $\phi$ can be extended to $\left\{v_{i+1}, \ldots, v_{n}\right\}$.

By Claim 3.4, if $T_{n}$ cannot be embedded into $G$, then $K$ is a component with $n-1$ vertices, contradicting that $G$ is a connected graph with at least $n$ vertices. So $T_{n}$ can be embedded into $G$ in this case.

Case 1.3. For each vertex $u$ in $N\left(u_{i-1}\right) \backslash K_{i},\left|K_{i} \backslash N(u)\right|=0$.

Take a vertex, denote by $u_{i}$, in $N\left(u_{i-1}\right) \backslash K_{i}$, and let $\phi\left(v_{i}\right)=u_{i}$.

If $d\left(u_{i}\right) \geq n-1$, since $u_{1}, u_{3} \notin N\left(u_{1}\right)$, by Lemma 2.2 (iv), $\phi$ can be extended to an embedding on $\left\{v_{1}, \ldots, v_{n-1}\right\}$. Since $d\left(u_{i}\right) \geq n-1$ and $\phi\left(v_{i}\right)=u_{i} \notin N\left(u_{i}\right)$, there is at least one free vertex in $N\left(u_{i}\right) \backslash\left\{\phi\left(v_{1}\right), \ldots, \phi\left(v_{n-1}\right)\right\}$ to embed $v_{n}$.

If $d\left(u_{i}\right)=n-2$, then $\left|N\left[u_{i}\right]\right|=n-1$. We claim that $\cup_{u \in N\left[u_{i}\right]} N[u]=N\left[u_{i}\right]$ if $T_{n}$ cannot be embedded into $G$. If there exists a vertex, denote by $u_{n-1}$, in $N\left(u_{1}\right) \backslash N\left[u_{i}\right]$, let $\phi\left(v_{i}\right)=u_{i}$ and extend $\phi$ to an embedding on $\left\{v_{1}, \ldots, v_{n-1}\right\}$ such that we embed some $v_{a} \in\left\{v_{i+1}, \ldots, v_{n-1}\right\}$ to $u_{n-1}$ (This is always possible since we can always embed $v_{n-1}$ to $u_{n-1}$ if $u_{n-1}$ is not previously used). Now $u_{i}, u_{n-1} \notin N\left(u_{i}\right)$, therefore there exists one free vertex in $N\left(u_{i}\right)$ to embedded $v_{n}$ and $T_{n}$ can be embedded into $G$. So we have shown that $N\left[u_{1}\right] \subseteq N\left[u_{i}\right]$. Assume that $\cup_{i=1}^{t} N\left[u_{i}\right] \subseteq N\left[u_{i}\right]$ for $1 \leq t \leq i-1$, we show that $N\left[u_{t+1}\right] \subseteq N\left[u_{i}\right]$. If there exists a vertex $u_{t}^{\prime} \in N\left[u_{t+1}\right] \backslash N\left[u_{i}\right]$, by Lemma 2.4 there exists a path $u_{t}^{\prime} u_{t-1}^{\prime} \ldots u_{1}^{\prime}$ outside $N\left[u_{i}\right]$. Reassign $\phi\left(v_{\alpha}\right)=u_{\alpha}^{\prime}$ for $1 \leq \alpha \leq t$. Since $u_{i} \notin N\left(\phi\left(v_{1}\right)\right)$, by Lemma 2.2 (iv), $\phi$ can be extended to an embedding on $\left\{v_{1}, \ldots, v_{n-1}\right\}$. Since $d\left(u_{i}\right)=n-2$ and $\phi\left(v_{i}\right)=u_{i}$, $\phi\left(v_{a}\right) \notin N\left(u_{i}\right)$ for $1 \leq a \leq t$, there is at least one free vertex in $N\left(u_{i}\right) \backslash\left\{\phi\left(v_{1}\right), \ldots, \phi\left(v_{n-1}\right)\right\}$ to embed $v_{n}$. Therefore we have shown that $N\left[K_{i}\right] \subseteq N\left[u_{i}\right]$. If there exist vertices $u^{\prime}, u^{\prime \prime}$ such that $u^{\prime} \in N\left(u_{i}\right) \backslash K_{i}$ and $u^{\prime \prime} \in N\left[u^{\prime}\right] \backslash N\left[u_{i}\right]$, since $d\left(u_{3}\right) \geq n-3,\left|N\left[u_{i}\right]\right|=n-1$ and $u_{1}, u_{3} \notin N\left(u_{3}\right)$ and $N\left(u_{3}\right) \subseteq N\left[u_{i}\right]$, $u_{3} u^{\prime} \in E(G)$. Reassign $\phi\left(v_{2}\right)=u^{\prime}$ and $\phi\left(v_{1}\right)=u^{\prime \prime}$. Since $u_{i} \notin N\left(\phi\left(v_{1}\right)\right)$, by Lemma 2.2 (iv), $\phi$ can be extended to $\left\{v_{1}, v_{2}, \ldots, v_{n-1}\right\}$. Since $u^{\prime \prime} u_{i} \notin E(G)$ and $d\left(u_{i}\right)=n-2$, there exists a free vertex in $N\left(u_{i}\right) \backslash\left\{\phi\left(v_{1}\right), \ldots, \phi\left(v_{n-1}\right)\right\}$ to embed $v_{n}$ and $T_{n}$ can be embedded into $G$. So we have shown that $\cup_{u \in N\left[u_{i}\right]} N[u]=N\left[u_{i}\right]$ if $T_{n}$ cannot be embedded into $G$. Therefore $N\left[u_{i}\right]$ is a component of $n-1$ vertices, contradicting that $G$ is a connected graph with at least $n$ vertices.

Now we discuss $d\left(u_{i}\right)=n-3$. Since $u_{1} u_{3} \notin E(G)$ and $d\left(u_{3}\right) \geq n-3$, there exists a vertex, denoted by $u_{2}^{\prime}$, in $N\left(u_{3}\right) \backslash N\left[u_{i}\right]$. If there exists a vertex $u_{1}^{\prime} \in N\left(u_{2}^{\prime}\right) \backslash N\left[u_{i}\right]$, then reassign $\phi\left(v_{2}\right)=u_{2}^{\prime}$ and $\phi\left(v_{1}\right)=u_{1}^{\prime}$. Since $\phi\left(v_{1}\right), \phi\left(v_{2}\right) \notin N\left(\phi\left(v_{i}\right)\right)$, by Lemma 2.2 (iv), $\phi$ can be extended to $\left\{v_{1}, \ldots, v_{n}\right\}$ and $T_{n}$ can be embedded into $G$. So $N\left(u_{2}^{\prime}\right) \subseteq N\left[u_{i}\right]$. Since $u_{2}^{\prime} u_{i} \notin E(G)$ and $d\left(u_{2}^{\prime}\right) \geq n-3, N\left(u_{2}^{\prime}\right)=N\left(u_{i}\right)$. Since $u_{2}^{\prime} u_{1}, u_{2}^{\prime} u_{3} \in E(G)$, we can reassign $\phi\left(v_{2}\right)=u_{2}^{\prime}$. Now $u_{i}$ has a non-neighbor in $K_{i}$. Note that $u_{3} \notin N\left(\phi\left(v_{1}\right)\right)$ still holds. This situation is exactly Case 1.2 we have proved.

Case 2. $|P|=5$.

In this case, $P=v_{n-1} v_{1} v_{2} v_{3} v_{n}$. If $d_{T_{n}}\left(v_{2}\right) \geq 3$, then there exists a vertex $v_{4}$ connected to $v_{2}$ (see Figure 22. If $d_{T_{n}}\left(v_{2}\right)=2$, then $N_{T_{n}}\left(v_{2}\right)=\left\{v_{1}, v_{3}\right\}$. If $T_{n}=P$, since $|V(G)| \geq 5$ and $\delta(G) \geq 2$, by Lemma 2.3, $P=T_{n}$ can be embedded in $G$. So if $d_{T_{n}}\left(v_{2}\right)=2$, we may assume that $d_{T_{n}}\left(v_{1}\right) \geq 3$ or $d_{T_{n}}\left(v_{3}\right) \geq 3$ (see Figure 3 . .

Let $u_{3}^{\prime}$ be a vertex with maximum degree in $V(G)$. If $d\left(u_{3}^{\prime}\right) \geq n-2$, if there exists a vertex $u_{2}^{\prime} \in N\left(u_{3}^{\prime}\right)$ such that there exists a vertex $u_{1}^{\prime} \in N\left(u_{2}^{\prime}\right) \backslash N\left[u_{3}^{\prime}\right]$, then let $\phi\left(v_{1}\right)=u_{1}^{\prime}, \phi\left(v_{2}\right)=u_{2}^{\prime}$ and $\phi\left(v_{3}\right)=u_{3}^{\prime}$. Since $u_{1}^{\prime}, u_{3}^{\prime} \notin N\left(u_{1}^{\prime}\right)$ and $\delta(G) \geq n-3$ and $d\left(u_{3}^{\prime}\right) \geq n-2$, by Lemma 2.2 (iv), $\phi$ can be extended to $\left\{v_{4}, \ldots, v_{n}\right\}$. If there is no such vertex $u_{2}^{\prime}$ in $N\left(u_{3}^{\prime}\right)$, then $N\left[u_{3}^{\prime}\right]$ is a component, so $d\left(u_{3}^{\prime}\right) \geq n-1$. 


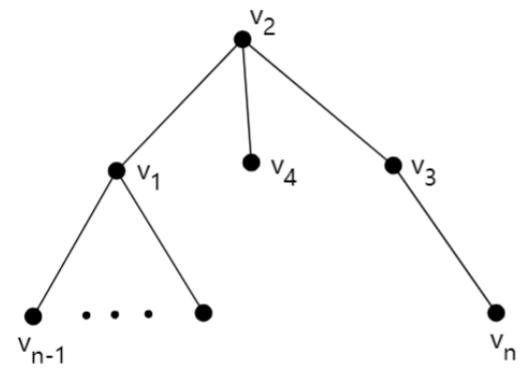

Figure 2: Case $d_{T_{n}}\left(v_{2}\right) \geq 3$

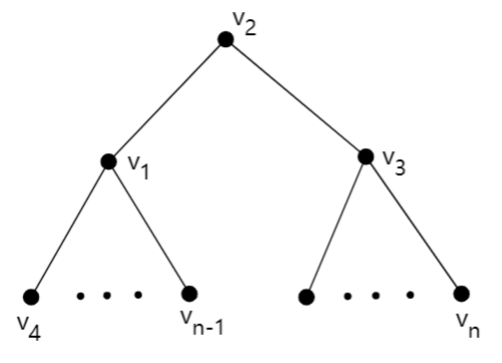

Figure 3: Case $d_{T_{n}}\left(v_{2}\right)=2$

If there exists a vertex $u_{1}^{\prime} \in N\left(u_{3}^{\prime}\right)$ such that $d\left(u_{1}^{\prime}\right) \geq n-2$, let $\phi\left(v_{1}\right)=u_{1}^{\prime}$ and $\phi\left(v_{3}\right)=u_{3}^{\prime}$ and $\phi\left(v_{2}\right) \in N\left(u_{1}^{\prime}\right)$, then by Lemma 2.2 (iv), $\phi$ can be extended to $\left\{v_{4}, \ldots, v_{n}\right\}$. So we may assume that $d\left(u_{x}\right)=n-3$ for all $u_{x} \in N\left(u_{3}^{\prime}\right)$. Since $\left|N\left[u_{3}^{\prime}\right]\right| \geq n$, for any vertex $u_{3}^{\prime \prime} \in N\left(u_{3}^{\prime}\right)$, we can find vertices $u_{1}^{\prime \prime}, u_{4}^{\prime \prime} \in N\left(u_{3}^{\prime}\right) \backslash N\left[u_{3}^{\prime \prime}\right]$. Let $\phi\left(v_{1}\right)=u_{1}^{\prime \prime}$ and $\phi\left(v_{2}\right)=u_{3}^{\prime}$ and $\phi\left(v_{3}\right)=u_{3}^{\prime \prime}$ and $\phi\left(v_{4}\right)=u_{4}^{\prime \prime}$. Since $\phi\left(v_{1}\right), \phi\left(v_{3}\right) \notin N\left(\phi\left(v_{1}\right)\right), d\left(\phi\left(v_{1}\right)\right) \geq n-3$ and $\phi\left(v_{1}\right), \phi\left(v_{3}\right), \phi\left(v_{4}\right) \notin N\left(\phi\left(v_{3}\right)\right)$, by Lemma 2.2 (iv), $\phi$ can be extended to $\left\{v_{4}, \ldots, v_{n}\right\}$ and $T_{n}$ can be embedded into $G$. Therefore, it is sufficient to consider that $G$ is an $(n-3)$-regular connected graph.

Case 2.1. $G$ is an $(n-3)$-regular connected graph with at least $n$ vertices and $d_{T_{n}}\left(v_{2}\right) \geq 3$ (see Figure 2p.

Claim 3.5 Let $G$ be $(n-3)$-regular and $d_{T_{n}}\left(v_{2}\right) \geq 3$ (see Figure 2). If there exist vertices $u_{x}, u_{y}, u_{a}, u_{b} \in$ $V(G)$ such that $u_{x} u_{y} \in E(G)$ and $u_{a}, u_{b} \in N\left(u_{y}\right) \backslash N\left[u_{x}\right]$ (see Figure 4), then $T_{n}$ can be embedded into $G$.

Proof. Let $\phi\left(v_{1}\right)=u_{a}, \phi\left(v_{2}\right)=u_{y}, \phi\left(v_{3}\right)=u_{x}$ and $\phi\left(v_{4}\right)=u_{b}$. Since $G$ is $(n-3)$-regular, $\phi\left(v_{1}\right), \phi\left(v_{2}\right) \notin$ $N\left(\phi\left(v_{1}\right)\right)$, and $\phi\left(v_{1}\right), \phi\left(v_{4}\right), \phi\left(v_{3}\right) \notin N\left(\phi\left(v_{3}\right)\right)$, by Lemma 2.2 (iv), $\phi$ can be extended to $\left\{v_{5}, \ldots, v_{n}\right\}$.

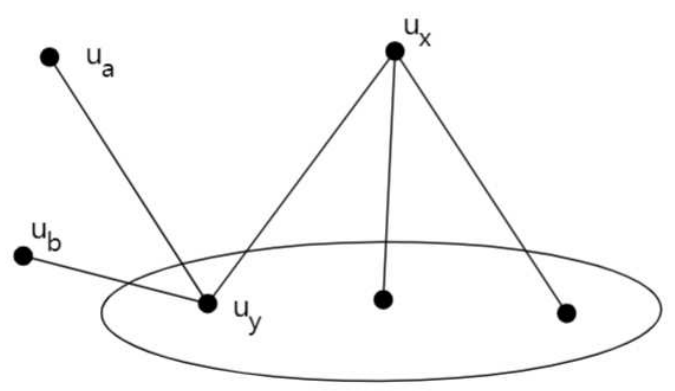

Figure 4: Figure for Claim 3.5

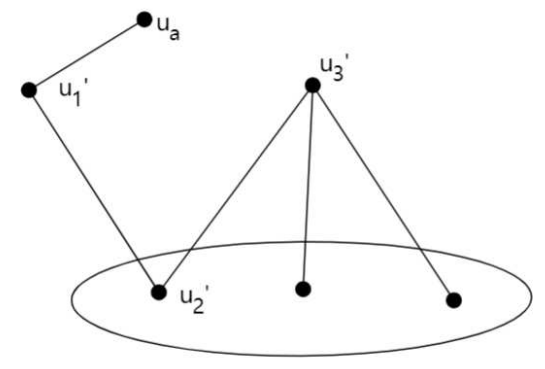

Figure 5: Figure for Case 2.1

Take a vertex $u_{3}^{\prime} \in V(G)$. Since $\left|N\left[u_{3}^{\prime}\right]\right|=n-2, N\left[u_{3}^{\prime}\right]$ is not a component. So there exists vertices $u_{1}^{\prime}, u_{2}^{\prime}$ such that $u_{2}^{\prime} \in N\left(u_{3}^{\prime}\right)$ and $u_{1}^{\prime} \in N\left(u_{2}^{\prime}\right) \backslash N\left[u_{3}^{\prime}\right]$ (see Figure 5). By Claim 3.5, $N\left[u_{2}^{\prime}\right] \subseteq N\left[u_{3}^{\prime}\right] \cup\left\{u_{1}^{\prime}\right\}$. If $u_{1}^{\prime}$ has two neighbors outside $N\left[u_{3}^{\prime}\right]$, then these two neighbors are outside $N\left[u_{2}^{\prime}\right]$. By Claim 3.5. $T_{n}$ 
can be embedded into $G$. If $N\left(u_{1}^{\prime}\right) \subseteq N\left[u_{3}^{\prime}\right]$, then $N\left(u_{1}^{\prime}\right)=N\left(u_{3}^{\prime}\right)$. If $T_{n}$ cannot be embedded into $G$, Claim 3.5 implies that $\cup_{u \in N\left[u_{3}^{\prime}\right]} N(u) \cup N\left(u_{1}^{\prime}\right)=N\left[u_{3}^{\prime}\right] \cup\left\{u_{1}^{\prime}\right\}$. Otherwise there exists a vertex $u \in N\left(u_{3}^{\prime}\right)$ such that $u$ has a neighbor $w$ outside $N\left[u_{3}^{\prime}\right] \cup\left\{u_{1}^{\prime}\right\}$, then $u$ has two neighbors $w$ and $u_{1}^{\prime}$ outside $N\left[u_{3}^{\prime}\right]$, contradicting Claim 3.5. Thus $N\left[u_{3}^{\prime}\right] \cup\left\{u_{1}^{\prime}\right\}$ is a component on $n-1$ vertices, contradicting that $G$ is a connected graph with at least $n$ vertices. Therefore, there exists exactly one vertex $u_{a} \in N\left(u_{1}^{\prime}\right) \backslash N\left[u_{3}^{\prime}\right]$. By Claim 3.5. $N\left(u_{1}^{\prime}\right)$ and $N\left(u_{a}\right)$ differ by at most one vertex. So $N\left(u_{1}^{\prime}\right) \subseteq N\left(u_{3}^{\prime}\right) \cup\left\{u_{a}\right\}$. If there exists a vertex $u \in N\left(u_{3}^{\prime}\right) \cap N\left(u_{1}^{\prime}\right)$ connected to $u_{a}$, then both $u_{a}$ and $u_{1}^{\prime}$ are in $N(u) \backslash N\left(u_{3}^{\prime}\right)$, contradicting Claim 3.5. So $N\left(u_{1}^{\prime}\right)$ and $N\left(u_{a}\right)$ have no common neighbor in $N\left[u_{3}^{\prime}\right]$, contradicting Claim 3.5 again. So $T_{n}$ can be embedded into $G$.

Case 2.2. $G$ is $(n-3)$-regular and $d_{T_{n}}\left(v_{2}\right)=2$ (see Figure 3), i.e. $T_{n}=T(p, n-3-p)$ for some $p$.

Claim 3.6 Let $G$ be an (n-3)-regular connected graph on at least $n$ vertices. If there exists $u_{1}^{\prime}, u_{2}^{\prime}, u_{3}^{\prime} \in$ $V(G)$ such that $u_{1}^{\prime} u_{2}^{\prime}, u_{2}^{\prime} u_{3}^{\prime} \in E(G)$ and $u_{1}^{\prime} u_{3}^{\prime} \notin E(G)$. Then $T_{n}$ can be embedded into $G$ or $N\left(u_{1}^{\prime}\right)=$ $N\left(u_{3}^{\prime}\right)$.

Proof. Suppose that $N\left(u_{1}^{\prime}\right) \neq N\left(u_{3}^{\prime}\right)$. Since $G$ is regular, there exists a vertex $u_{4} \in N\left(u_{1}^{\prime}\right) \backslash N\left[u_{3}^{\prime}\right]$. Let $\phi\left(v_{1}\right)=u_{1}^{\prime}, \phi\left(v_{2}\right)=u_{2}^{\prime}, \phi\left(v_{3}\right)=u_{3}^{\prime}$ and $\phi\left(v_{4}\right)=u_{4}$. Since $u_{1}^{\prime} u_{3}^{\prime} \notin E(G)$, and $u_{1}^{\prime}, u_{3}^{\prime}, u_{4} \notin N\left(u_{3}^{\prime}\right)$, by Lemma 2.2 (iv), $\phi$ can be extended to an embedding of $T_{n}$.

Assume that $T_{n}$ cannot be embedded into $G$. Let $A_{1}$ be a maximal set in $G$ satisfying that it is an independent set and its any two vertices have a common neighbor. By Claim 3.6, all vertices in $A_{1}$ have the same set of neighbors, say $B$. Then $|B|=n-3$. We claim that $V(G)=A_{1} \cup B$. Otherwise, there exists a vertex $u_{x} \in V(G) \backslash\left(A_{1} \cup B\right)$ such that $u_{x}$ is connected to a vertex in $B$ and none of the vertices in $A_{1}$ is adjacent to $u_{x}$. By Claim 3.6, $N\left(u_{x}\right)=B$. This contradicts the maximality of $A_{1}$. Since it holds for all such maximal sets, we can divide $B$ into $k$ sets $B_{1}, B_{2}, \ldots, B_{k}$ such that $B_{j}$ is such a maximal set for each $j \in[k]$. Since $G$ is regular, $\left|A_{1}\right|=\left|B_{1}\right|=\ldots=\left|B_{k}\right|=a, n-3=a k$ and $|V(G)|=n-3+a$ (See Figure 6). Since $|V(G)| \geq n, a \geq 3$.

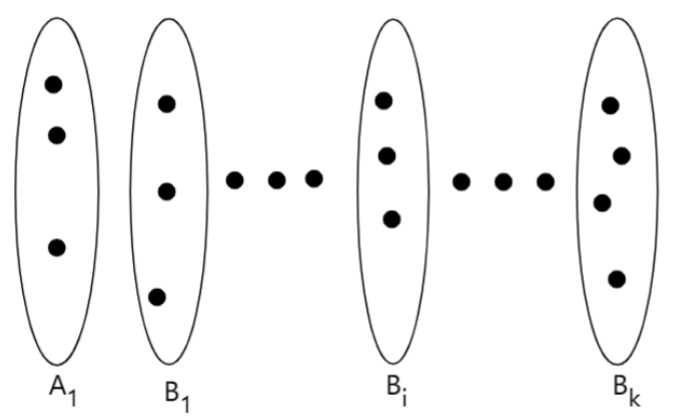

Figure 6: $K_{a, a, \ldots, a}$

If $k=1$, since $G$ is $(n-3)$-regular, $G=K_{n-3, n-3}$, and in this case, $T(p, n-3-p)$ cannot be embedded into $K_{n-3, n-3}$. This is because that $T(p, n-3-p)$ is a bipartite graph with 2 and $n-2$ vertices in each of the two parts. This is situation (i) in Theorem 1.2

If $k \geq 2$, then let $\phi\left(v_{1}\right) \in B_{1}, \phi\left(v_{2}\right) \in B_{2}$ and $\phi\left(v_{3}\right) \in A_{1}$. 


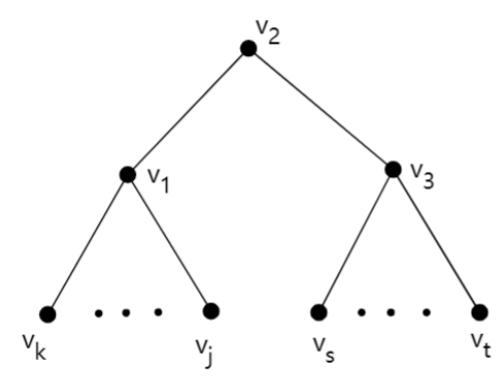

Figure 7: $T(p, n-3-p)(2 \leq p \leq n-5)$

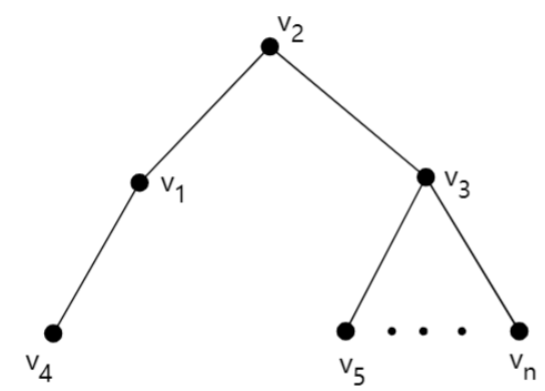

Figure 8: $T(1, n-4)$

If $d\left(v_{1}\right) \geq 3$ and $d\left(v_{3}\right) \geq 3$ (see Figure 7), let $\phi\left(v_{k}\right), \phi\left(v_{j}\right) \in A_{1}$ and $\phi\left(v_{s}\right), \phi\left(v_{t}\right) \in B_{1}$, then the other leaves can be embedded in $A \cup B$ since $G$ is a balanced complete $(k+1)$-partite graph, and $T_{n}$ can be embedded into $G$. Now consider $d\left(v_{1}\right)=2$ or $d\left(v_{3}\right)=2$, i.e. $T_{n}=T(1, n-4)$. We claim that $T(1, n-4)$ cannot be embedded into $G$. Without loss of generality, assume that $d\left(v_{1}\right)=2$ (see Figure 8). Suppose that $T(1, n-4)$ can be embedded into $G$ and $v_{3}$ is embedded into $A_{1}$ (without loss of generality). Then all $n-3$ vertices connected to $v_{3}$ should be embedded outside $A_{1}$. Since $\left|V(G) \backslash A_{1}\right|=n-3, \phi\left(v_{1}\right)$ must be in $A_{1}$. Since all vertices of $T(1, n-4) \backslash\left\{v_{1}, v_{3}\right\}$ are connected to $v_{1}$ or $v_{3}$, then every vertex in $T(1, n-4) \backslash\left\{v_{1}, v_{3}\right\}$ must be embedded outside $A_{1}$, i.e. there are $n-2$ vertices should be embedded outside $A_{1}$, but $\left|V(G) \backslash A_{1}\right|=n-3$. This is impossible. So in this case $T(1, n-4)$ cannot be embedded into $G$. This is situation (ii) in Theorem 1.2 .

Case 3. $|P|=4$.

In this case we label the vertices of $T_{n}$ as in Figure 9. Note that $\left\{v_{1}, v_{2}, \ldots, v_{n}\right\}$ is a conventional labelling. Since $d\left(v_{1}\right)+d\left(v_{2}\right)=n$ and $\Delta\left(T_{n}\right) \leq n-3, d\left(v_{1}\right), d\left(v_{2}\right) \geq 3$.

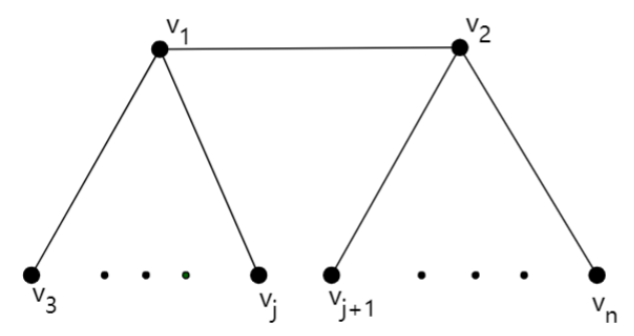

Figure 9: $T_{n}$ has n-2 leaves

Case 3.1. $\Delta(G) \geq n-1$.

Let $u, v \in V(G)$ such that $d(u)=\Delta(G)$ and $u v \in E(G)$. Embed $\phi\left(v_{2}\right)=u$ and $\phi\left(v_{1}\right)=v$. By Lemma 2.2 (iv), $\phi$ can be extended to $\left\{v_{1}, \ldots, v_{n-2}\right\}$. Recall that $v_{n-1}$ and $v_{n}$ have the same parent (see Figure 9). Since $d(u) \geq n-1$, there are two free vertices in $N(u) \backslash\left\{\phi\left(v_{1}\right), \ldots, \phi\left(v_{n-2}\right)\right\}$ to embed $v_{n-1}$ and $v_{n}$. So $T_{n}$ can be embedded in $G$.

Case 3.2. $\Delta(G)=n-2$. 
Let $\phi\left(v_{2}\right)=u_{2}^{\prime}$ such that $d\left(u_{2}^{\prime}\right)=n-2$. We claim that there exists a vertex $u_{1}^{\prime} \in N\left(u_{2}^{\prime}\right)$ such that there exists a vertex $u_{3}^{\prime} \in N\left(u_{1}^{\prime}\right) \backslash N\left[u_{2}^{\prime}\right]$. Otherwise $\cup_{u \in N\left[u_{2}^{\prime}\right]}=N\left[u_{2}^{\prime}\right]$. So $N\left[u_{2}^{\prime}\right]$ is a component with $n-1$ vertices, contradicting that $G$ is a connected graph on at least $n$ vertices. Let $\phi\left(v_{1}\right)=u_{1}^{\prime}$ and $\phi\left(v_{3}\right)=u_{3}^{\prime}$. By Lemma 2.2 (iv), $\phi$ can be extended to $\left\{v_{1}, \ldots, v_{n-2}\right\}$. Since $d\left(u_{2}^{\prime}\right)=n-2$ and $u_{3}^{\prime} \notin N\left(u_{2}^{\prime}\right)$, there are two free vertices in $N\left(u_{2}^{\prime}\right) \backslash\left\{\phi\left(v_{1}\right), \ldots, \phi\left(v_{n-2}\right)\right\}$ to embed $v_{n-1}$ and $v_{n}$. So $T_{n}$ can be embedded into $G$.

Case 3.3. $G$ is $(n-3)$-regular.

Claim 3.7 If there exists a vertex $u_{2}^{\prime} \in V(G)$ such that there exists a vertex $u_{1}^{\prime} \in N\left(u_{2}^{\prime}\right)$ with two neighbors $u_{x}, u_{y} \in N\left(u_{1}^{\prime}\right) \backslash N\left[u_{2}^{\prime}\right]$, then $T_{n}$ can be embedded into $G$.

Proof. Let $\phi\left(v_{1}\right)=u_{1}^{\prime}, \phi\left(v_{2}\right)=u_{2}^{\prime}, \phi\left(v_{3}\right)=u_{x}$ and $\phi\left(v_{4}\right)=u_{y}$. By Lemma 2.2 (iv), $\phi$ can be extended to $\left\{v_{1}, \ldots, v_{n-2}\right\}$. Since $u_{2}^{\prime}, u_{x}, u_{y} \notin N\left(u_{2}^{\prime}\right)$ and $d\left(u_{2}^{\prime}\right)=n-3$, there are two free vertices in $N\left(u_{2}^{\prime}\right) \backslash\left\{\phi\left(v_{1}\right), \ldots, \phi\left(v_{n-2}\right)\right\}$ to embed $v_{n-1}$ and $v_{n}$. So $T_{n}$ can be embedded into $G$.

Claim 3.8 If $T_{n}$ cannot be embedded into $G$, then for any vertex $u_{x} \in V(G)$, there exists a vertex $u_{y} \in N\left(u_{x}\right)$ such that $u_{y}$ has exactly one neighbor outside $N\left[u_{x}\right]$.

Proof. By Claim 3.7, any vertex in $N\left(u_{x}\right)$ has at most one neighbor outside $N\left[u_{x}\right]$. If $\cup_{u \in N\left[u_{x}\right]} N[u] \subseteq$ $N\left[u_{x}\right]$, then $N\left[u_{x}\right]$ is a component on $n-2$ vertices, a contradiction.

If $T_{n}$ cannot be embedded into $G$, let $u_{x} \in V(G)$ and $u_{y} \in N\left(u_{x}\right)$ such that $u_{z} \in N\left(u_{y}\right) \backslash N\left[u_{x}\right]$. Since $G$ is (n-3)-regular and $u_{z} \in N\left(u_{y}\right) \backslash N\left[u_{x}\right]$, there is exactly one vertex $u_{a} \in N\left(u_{x}\right) \backslash N\left(u_{y}\right)$ and $N\left(u_{y}\right)=N\left[u_{x}\right] \cup\left\{u_{z}\right\} \backslash\left\{u_{y}, u_{a}\right\}$. By Claim 3.7. $N\left[u_{y}\right] \backslash\left\{u_{x}\right\} \subseteq N\left[u_{z}\right]$. Since $u_{y} u_{a} \notin E(G),\left|d\left(u_{a}\right)\right|=n-3$ and $\left|N\left[u_{x}\right]\right|=n-2$, there is exactly one vertex $u_{b} \in N\left(u_{a}\right) \backslash N\left(u_{x}\right)$. If $u_{b}=u_{z}$, then $N\left(u_{z}\right)=N\left(u_{x}\right)$. For any vertex $u \in N\left(u_{x}\right), u_{z} \in N(u) \backslash N\left(u_{x}\right)$. Then by Claim 3.7, $N\left[u_{x}\right] \cup\left\{u_{z}\right\}$ is a component on $n-1$ vertices, a contradiction. So $u_{b} \neq u_{z}$. Since $u_{a}$ has exactly one neighbor outside $N\left(u_{x}\right)$, $N\left[u_{a}\right]=N\left[u_{x}\right] \cup\left\{u_{b}\right\} \backslash\left\{u_{y}\right\}$. Since all vertices in $N\left(u_{x}\right)$ have at most one neighbor outside $N\left(u_{x}\right)$ and $u_{z}$ is a neighbor of all vertices in $N\left(u_{x}\right) \backslash\left\{u_{a}\right\}=N\left(u_{a}\right) \backslash\left\{u_{b}, u_{x}\right\} \cup\left\{u_{y}\right\}$, by Claim 3.7 $u_{b}$ is not adjacent to any of these vertices, i.e, $u_{b}$ has no neighbor in $N\left(u_{a}\right) \backslash\left\{u_{b}\right\}$, contradicting Claim 3.7 again. So $T_{n}$ can be embedded into $G$ in this case.

\section{Tree-star Ramsey number}

In this section, we apply Theorem 1.2 to obtain the Ramsey number of a tree versus a star for some cases.

Proof of Theorem 1.3. For any red-blue-coloring of $E\left(K_{m+n-3}\right)$, let $G_{R}$ and $G_{B}$ be the red graph and the blue graph respectively. If $\Delta\left(G_{B}\right) \geq m$, then there is a blue $K_{1, m}$. So we may assume that $\Delta\left(G_{B}\right) \leq m-1$. Hence $\delta\left(G_{R}\right) \geq(m+n-4)-(m-1)=n-3$. Since $m+n-3$ is not a linear combination of $n-1$ and $n-2$, then $G_{R}$ contains a component with order at least $n$. By Theorem 1.2 . $T_{n}$ can be embedded into $G_{R}$.

Fact 4.1 For positive integers $m$ and $n, m+n-4$ is a linear combination of $n-1$ and $n-2$ and $m+n-3$ is not a linear combination of $n-1$ and $n-2$ if and only if $m=k(n-1)+3$ and $0 \leq k \leq n-5$. 
Proof of Fact 4.1. Necessity: If $m+n-4=k(n-1)+l(n-2), k \geq 0$ and $l \geq 1$, then

$$
m+n-3=k(n-1)+l(n-2)+(n-1)-(n-2)=(k+1)(n-1)+(l-1)(n-2),
$$

contradicting that $m+n-3$ is not a linear combination of $n-1$ and $n-2$. Therefore $l=0$ and $m=(k-1)(n-1)+3$, where $k \geq 1$. Let us rewrite $m=k(n-1)+3$, where $k \geq 0$. If $k \geq n-4$, then

$$
m+n-3=k(n-1)+n=(k-n+4)(n-1)+(n-2)(n-2),
$$

and $m+n-3$ is a linear combination of n- 1 and $\mathrm{n}-2$. So $0 \leq k \leq n-5$.

Sufficiency: If $m=k(n-1)+3$ and $0 \leq k \leq n-5$, then $m+n-4=(k+1)(n-1)$ is a linear combination of $n-1$ and $n-2$. If $m+n-3$ is a linear combination of $n-1$ and $n-2$, then $m+n-3=k(n-1)+n=k^{\prime}(n-1)+l^{\prime}(n-2)\left(k^{\prime}, l^{\prime} \geq 0\right)$. So $\left(k+2-k^{\prime}\right)(n-1)=\left(l^{\prime}+1\right)(n-2)$. Since $n-1$ is relatively prime to $n-2, k+2-k^{\prime}=c(n-2)$ for $c \geq 1$. Therefore $k \geq(n-2)-2+k^{\prime} \geq n-4$, a contradiction.

For a positive integer $a$ dividing $n-3$, let $B(n-3+a)$ be the balanced complete $\frac{n-3+a}{a}$-partite graph on $n-3+a$ vertices.

Proof of Theorem 1.4. Since $m+n-4=(k+1)(n-1)$, we can partition $V\left(K_{m+n-4}\right)$ into $k+1$ disjoint equal parts with $n-1$ vertices in each part. Color edges in each part red and other edges blue. Then $\Delta\left(G_{B}\right) \leq m+n-4-(n-1)=m-3$. So this coloring yields neither a red $T_{n}$ nor a blue $K_{1, m}$. Therefore $R\left(T_{n}, K_{1, m}\right) \geq m+n-3$.

(i). By Fact 4.1 and Theorem $1.3, R\left(T_{n}, K_{1, m}\right) \leq m+n-3$. Therefore $R\left(T_{n}, K_{1, m}\right)=m+n-3$.

(ii). For any red-blue-coloring of $E\left(K_{m+n-3}\right)$, let $G_{R}$ and $G_{B}$ be the red graph and the blue graph respectively. If $\Delta\left(G_{B}\right) \geq m$, then there is a blue $K_{1, m}$. So we may assume that $\Delta\left(G_{B}\right) \leq m-1$. Hence $\delta\left(G_{R}\right) \geq(m+n-4)-(m-1)=n-3$. By Fact 4.1. $G_{R}$ contains a component with at least $n$ vertices. By Theorem 1.2 if $m+n-3$ is not a linear combination of $n-1, n-2, n-3+a_{1}, \ldots$, $n-3+a_{d}$, then $T(1, n-4)$ can be embedded into $G_{R}$. So $R\left(T(1, n-4), K_{1, m}\right) \leq m+n-3$. Therefore $R\left(T(1, n-4), K_{1, m}\right)=m+n-3$.

If $m+n-3=k_{1}(n-1)+l_{1}(n-2)+s_{1}\left(n-3+a_{1}\right)+\ldots+s_{d}\left(n-3+a_{d}\right)$, for red-blue-coloring of $E\left(K_{m+n-3}\right)$, let $G_{R}$ and $G_{B}$ be the red graph and the blue graph respectively. Let $G_{R}=k_{1} K_{n-1} \cup$ $l_{1} K_{n-2} \cup s_{1} B\left(n-3+a_{1}\right) \cup \ldots \cup s_{d} B\left(n-3+a_{d}\right)$ and $G_{B}=G_{R}^{c}$. By Theorem $1.2 T(1, n-4)$ cannot be embedded into $G_{R}$. Since $\delta\left(G_{R}\right) \geq n-3$, then $\Delta\left(G_{B}\right) \leq(m+n-4)-(n-3)=m-1$. So there is neither a red $T_{n}$ nor a blue $K_{1, m}$. Therefore $R\left(T(1, n-4), K_{1, m}\right) \geq m+n-2$. The same lower bound was given in [6]. So $R\left(T(1, n-4), K_{1, m}\right)=m+n-2$.

(iii). For any red-blue-coloring of $E\left(K_{m+n-3}\right)$, let $G_{R}$ and $G_{B}$ be the red graph and the blue graph respectively. If $\Delta\left(G_{B}\right) \geq m$, then there is a blue $K_{1, m}$. So we may assume that $\Delta\left(G_{B}\right) \leq m-1$. Hence $\delta\left(G_{R}\right) \geq n-3$. By Fact 4.1 and $m+n-3$ is not a linear combination of $2 n-6, n-1$ and $n-2$, there exists a component $G^{\prime}$ such that $n \leq\left|V\left(G^{\prime}\right)\right| \neq 2 n-5$. By Theorem 1.2 . $T(p, n-3-p)$ can be embedded into $G^{\prime}$. Therefore $R\left(T(p, n-3-p), K_{1, m}\right)=m+n-3$. If $m+n-3=r(2 n-6)+s(n-1)+t(n-2)$, then we can partition $K_{m+n-3}$ into $r+s+t$ disjoint parts such that $r$ parts have order $2 n-6$ and $s$ parts have order $n-1$ and $t$ parts have order $n-2$. Let $G_{R}=r K_{n-3, n-3} \cup s K_{n-1} \cup t K_{n-2}$ and $G_{B}=G_{R}^{c}$. Then $\Delta\left(G_{B}\right) \leq m+n-4-(n-3)=m-1$ and $T(p, n-3-p)$ cannot be embedded into $G$. Therefore $R\left(T(p, n-3-p), K_{1, m}\right) \geq m+n-2$. The same lower bound was given in [6]. So 
$R\left(T(1, n-4), K_{1, m}\right)=m+n-2$.

Acknowledgement The research is supported in part by National Natural Science Foundation of China (No. 11931002).

\section{References}

[1] J. A. Bondy and U. S. R. Murty, Graph theory with applications, The Macmillan Press Ltd., 1976.

[2] S. A. Burr: Generalized Ramsey theory for graphs - a survey, Graphs and Combinatorics, Lecture Notes in Mathematics, 406(1974), 52-75.

[3] E. J. Cockayne: Some tree-star ramsey numbers, J. Combinatorial Theory, Ser. B, 17(1974), 183-187.

[4] G. A. Dirac: Some theorems on abstract graphs, Proc. London Math. Soc., 2(1952), 69-81.

[5] P. Erdős, Z. Füredi, M. Loebl, and V. T. Sós: Discrepency of trees, Studia Sci. Math. Hungra, 30(1995), 47-57.

[6] Y. Guo and L. Volkmann: Tree-Ramsey numbers, Australasian J. Combin., 11(1995), 169-175.

[7] F. Havet, B. Reed, M. Stein, and D. Wood: A variant of the Erdős and Sós conjecture, Journal of Graph Theory, 94(2019), 131-158.

[8] T. D. Parsons: Path-star ramsey numbers, J. Combinatorial Theory, Ser. B, 17(1974), 51-58. 